\title{
Percepción de Estudiantes de Pedagogía respecto de la Autorregulación del Aprendizaje
}

\author{
Carolina Zambrano(1)*, Felipe Albarran ${ }^{(2)}$ y Pedro A. Salcedo(1) \\ (1) Departamento de Metodología de Investigación e Informática Educacional, Facultad de Educación \\ Universidad de Concepción, Concepción, Chile. (e-mail: carolinazambrano@gmail.com) \\ (2) Facultad de Educación, Universidad de Concepción, Concepción, Chile.
}

${ }^{*}$ Autor a quien debe ser enviada la correspondencia

Recibido Oct. 25, 2017; Aceptado Dic. 7, 2017; Versión final Ene. 10, 2018, Publicado Jun. 2018

\begin{abstract}
Resumen
En el estudio presentado en este artículo se ha descrito y analizado qué comprenden los estudiantes por autorregulación del aprendizaje, qué hacen al aplicar las distintas fases de este proceso y cuáles son las dificultades que tienen para regular su aprendizaje. Para analizar las respuestas de los estudiantes en relación con el proceso de autorregulación del aprendizaje se utiliza el modelo de Zimmerman con el objetivo de poder comparar las percepciones recogidas versus el modelo propuesto por dicho autor. La muestra estuvo compuesta por 60 estudiantes de pedagogía de una universidad en Chile. Los principales hallazgos muestran que los estudiantes relacionan el concepto de autorregulación del aprendizaje principalmente con la organización general previa al estudio y con la regulación de sus emociones. Respecto al proceso de autorregulación del aprendizaje se concluye que la fase de planificación y ejecución son incipientes, y se discuten sus causas y efectos.
\end{abstract}

Palabras clave: autorregulación del aprendizaje; deserción académica; planificación del estudio; proceso de aprendizaje

\section{Perception of Pedagogy Students regarding Self-Regulation of Learning}

\begin{abstract}
In the study presented in this article it is described and analyzed what students understand by self-regulation of learning, what do the when applying the different phases of this process and what are the difficulties they have to do to regulate their learning. To analyze the students' answers in relation to the process of selfregulation of learning, the Zimmerman model is used in order to compare the perceptions collected versus the model proposed by the author. The sample consisted of 60 students of pedagogy from a university in Chile. The main findings show that students relate the concept of self-regulation of learning mainly with the general organization prior to the study and with the regulation of their emotions. Regarding the process of selfregulation of learning, it is concluded that the planning and execution phase are incipient, and their causes and effects are discussed.
\end{abstract}




\section{INTRODUCCIÓN}

El sistema educacional chileno ha vivido un proceso de expansión y transformación de la educación superior, lo que ha generado un ingreso masivo de estudiantes que produce, entre otras consecuencias, la deserción estudiantil, principalmente producto de que estos no logran adecuarse a las exigencias de la vida universitaria. En este sentido, autores como Donoso y, Donoso y Arias (2010) han estudiado la evolución del sistema educacional en Chile a través de los procesos de cambios de oferta y demanda, cuyos resultados reflejan cómo este fenómeno ha derivado en la necesidad de generar estrategias orientadas a la retención de estudiantes en las diversas universidades. Además, otro factor que ha influido en el sistema educacional universitario corresponde al creciente posicionamiento del enfoque por competencias que ha sido promovido por entidades internacionales, tales como, el Banco Mundial, la Organización para la Cooperación y el Desarrollo Económico (OCDE), el Banco Iberoamericano, y la influencia europea del Proceso de Bolonia (Cuba-Esquivel, 2016). Así, en este escenario de acceso masivo de estudiantes en la educación superior y del enfoque por competencias en los curriculum universitarios es que se requiere capacidad de autonomía en los estudiantes universitarios, lo que trae por consecuencia, la necesidad de comprender y evaluar cómo los estudiantes autorregulan su aprendizaje (Díaz et. al, 2010; Pool-Cibrián y Martínez-Guerrero, 2013; Daura, 2015).

Por lo anterior, la siguiente pregunta es relevante ¿por qué es importante la autorregulación del aprendizaje? Porque es un constructo psicológico que ha sido estudiado en diversas investigaciones, demostrándose que es un factor predictor del logro académico (Boekaerts, Pintrich y Zeidner, 2005; Sitzmann y Ely, 2011). Y, por tanto, un factor que permite a las instituciones desarrollar políticas y acomodaciones curriculares que disminuyan la deserción estudiantil. Así mismo, es importante señalar que desde la perspectiva de las habilidades del siglo XXI (Trilling y Fadel, 2009) se sustenta la posibilidad de poder conocer qué comprenden los estudiantes de pedagogía respecto del concepto de autorregulación del aprendizaje y cómo describen aplicar el proceso de autorregulación del aprendizaje desde la perspectiva del modelo de Barry Zimmerman. Estudiar la habilidad de aprender a aprender (Trilling y Fadel, 2009) desde la autorregulación del aprendizaje se apoya en el hecho de que los futuros profesores serán quienes podrán promover este tipo de aprendizaje en el aula a sus estudiantes para fomentar en ellos las habilidades necesarias en la sociedad donde se desenvolverán.

Otra motivación para realizar el estudio tiene relación con la incipiente cantidad de investigaciones en la temática en estudiantes de pedagogía, situación que se evidencia en la revisión sistemática realizada por Hernández y Camargo (2017) quienes reportan que entre 2005 y 2015 se publicaron sólo 7 artículos donde los participantes son estudiantes de pedagogía. En este sentido, encontramos tres investigaciones que se enfocan en el estudiante de pedagogía utilizando el enfoque cualitativo para describir mediante estudios de caso el proceso de autorregulación del aprendizaje (Pranke y Bragagnolo-Frison, 2015; Garello y Rinaudo, 2013; Simão y Flores, 2010). No obstante, ninguno de los estudios anteriores es realizado con estudiantes de pedagogía en Chile.

El presente artículo da cuenta de los resultados de una investigación, de carácter preliminar, de tipo descriptiva que aborda la percepción que posee un grupo de estudiantes de pedagogía del concepto de autorregulación del aprendizaje y sobre el proceso de autorregulación del aprendizaje desde la perspectiva del modelo de Barry Zimmerman. En efecto, la investigación tiene por objetivo analizar y describir qué comprenden los estudiantes de pedagogía por el concepto de autorregulación del aprendizaje, qué hacen al aplicar las distintas fases de este proceso usando como referencia el modelo de autorregulación del aprendizaje de Zimmerman y describir cuáles son las dificultades que señalan al llevar a cabo dicho proceso.

\section{Autorregulación del Aprendizaje}

Para Zimmerman y Moylan el aprendizaje autorregulado es un proceso desarrollado por los estudiantes cuando estos tienen conciencia de sus propios procesos cognitivos, socioafectivos y motivacionales (Zimmerman y Moylan, 2009). En este contexto, se debería orientar al estudiante para que se cuestione, revise, planifique, controle y evalué sus propias acciones de aprendizaje (Pintrich, 1990).

En el ámbito de investigación sobre autorregulación del aprendizaje se distinguen varios modelos de autorregulación del aprendizaje. Seis fueron analizados según Panadero (2017) donde destacan: Zimmerman (2000), Pintrich (2000), Winne (1996, 1997), Boekaerts (1999) y Boekaerts y Niemivirta (2000). No obstante, en esta investigación se utiliza el modelo de Zimmerman en su última versión (Zimmerman y Moylan, 2009) debido a que este modelo ha sido el más extendido en la literatura científica en el ámbito de la psicología educacional (Panadero y Alonso-Tapia, 2014). Y por incluir un proceso de autorregulación cíclico que explica en detalle en la versión 2009 las tres fases de autorregulación del aprendizaje que se pueden considerar como un "modelo ideal" con el cual contrastar las percepciones de los estudiantes respecto de este proceso. 


\section{Modelo Cíclico de Zimmerman sobre autorregulación del aprendizaje}

El trabajo de Zimmerman tiene una base socio-cognitiva que explica la autorregulación del aprendizaje a través de un modelo cíclico. Este modelo ha evolucionado en el tiempo hacia un modelo más detallado y con un alto grado de penetración en la literatura científica (Panadero y Alonso-Tapia, 2014). El modelo tiene tres versiones: la primera del año 2000 (Zimmerman, 2000), la segunda del año 2003 (Zimmerman y Campillo, 2003) y la última del año 2009 (Zimmerman y Moylan, 2009). Cabe destacar que, en su última versión, éste incluye más procesos en la fase de ejecución y define de forma específica cada uno de ellos. Las tres fases del modelo propuesto en (Zimmerman y Moylan, 2009) se explican a continuación con ayuda de la Fig. 1, la que muestra la traducción del modelo realizado por Panadero y Alonso-Tapia (2014).

Fase de Planificación: es la fase inicial que se compone del proceso de "Análisis de la tarea y de las Creencias auto-motivadoras". Por ejemplo, cuando un estudiante se enfrenta por primera vez a una tarea, él debiese realizar dos procesos: 1 ) establecer los objetivos que debe alcanzar y 2) realizar una planificación estratégica. Estos dos procesos permiten llevar a cabo el análisis de la tarea. En el caso de las Creencias automotivadoras se establece que influyen cinco tipos de variables: 1) autoeficacia, 2) expectativas de resultado, 3) valor de la tarea, 4) interés y 5) orientación a metas. Estas variables son personales y permiten generar la motivación para realizar la actividad.

Fase de Ejecución: se compone de dos procesos: 1) auto-control y 2) auto-observación. El primero se define como el proceso para mantener la concentración y el interés a través de estrategias de tipo metacognitivo o de tipo motivacional. Por un lado, el autocontrol metacognitivo se establece al escoger una estrategia específica, por ejemplo, al hacer un resumen. Por otro lado, el autocontrol motivacional se refiere a incentivar el interés, por ejemplo, usando mensajes de recuerdo sobre la meta. El segundo proceso se define como la comparación entre lo que se está haciendo con respecto a un modelo ideal de ejecución.

Fase de Auto Reflexión: esta fase se compone del proceso auto-juicio y del proceso auto-reacción, los cuales interactúan entre sí. Por una parte, el auto-juicio es el proceso que permite al estudiante juzgar su ejecución. De este modo, el estudiante puede realizar una autoevaluación que le permite valorar su trabajo, basándose en los criterios de calidad que debieron ser establecidos claramente al inicio de la actividad por el profesor. También, el estudiante realizará atribuciones causales que implican el cómo se auto-explica el éxito o fracaso en la actividad. Por otra parte, el proceso de auto-reacción se refiere a las reacciones del estudiante ante sus auto-juicios. Así, su auto-reacción puede ser de satisfacción, afecto, adaptación o una reacción defensiva.

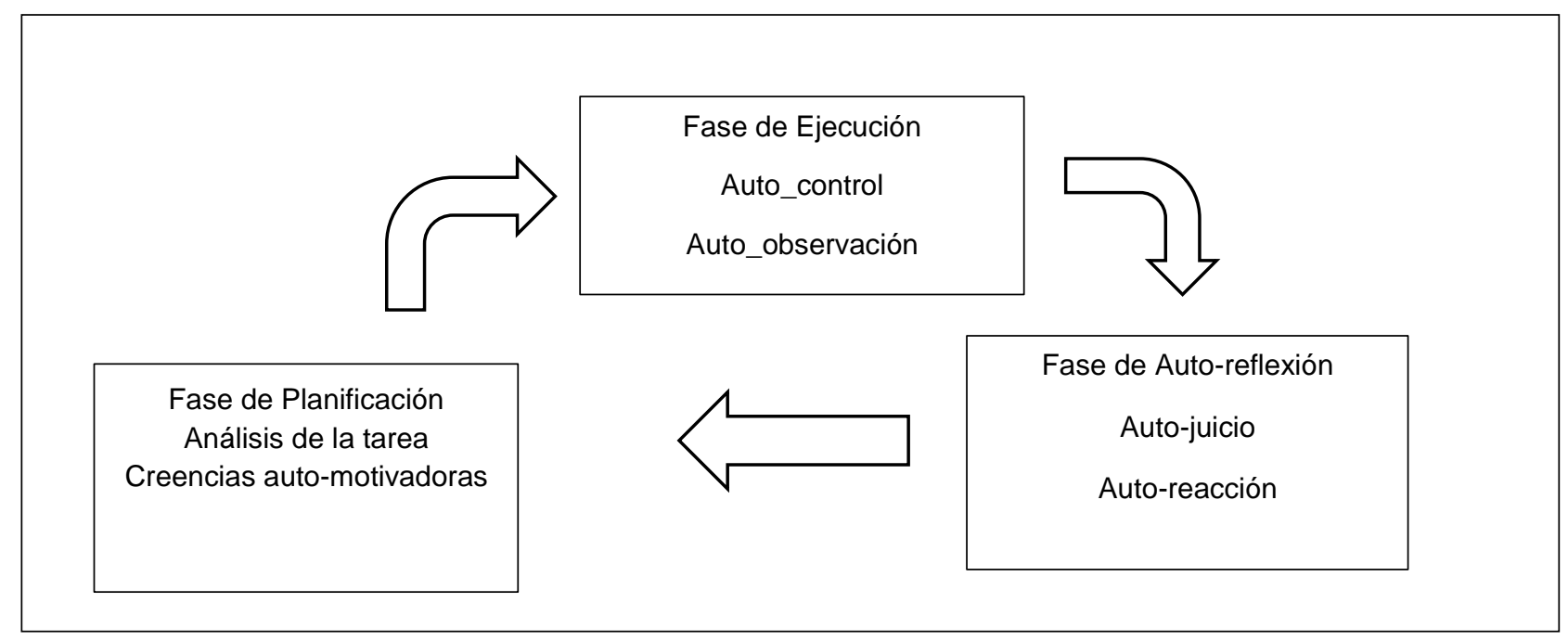

Fig. 1: Fases y procesos de autorregulación del aprendizaje según Zimmerman y Moylan (2009) Fuente: Zimmerman y Moylan (2009) traducido por Panadero y Alonso-Tapia (2014)

\section{METODO}

Para llevar a cabo esta investigación y comprender las percepciones de un grupo de estudiantes de pedagogía respecto del concepto de autorregulación del aprendizaje, el proceso de autorregulación del aprendizaje desde la perspectiva del modelo de Zimmerman y los agentes que dificultan la regulación de sus aprendizajes, se utilizó el enfoque de investigación cualitativo de acuerdo con Hernández, Fernández y Batista (2010) mediante un estudio de caso. 


\section{Diseño de investigación}

El diseño es no experimental de tipo transversal descriptivo, es decir, se tiene como objetivo categorizar y proporcionar una visión del fenómeno en estudio.

\section{Preguntas de investigación}

Las preguntas formuladas para guiar la investigación fueron:

1) ¿Qué comprenden (los estudiantes de pedagogía) sobre el concepto de autorregulación del aprendizaje?

2) ¿Qué describen hacer respecto de las distintas fases del proceso de autorregulación del aprendizaje desde la perspectiva del modelo de Zimmerman?

3) ¿Cuáles son los agentes anti regulación de su aprendizaje?

\section{Participantes}

Para la selección de la muestra se consideró la técnica de muestreo no probabilístico, dado que los participantes fueron seleccionados de forma intencionada. Los criterios para la selección fueron: a) cursar segundo, tercero o cuarto año de pedagogía y b) participar voluntariamente del estudio. Se realizaron seis entrevistas grupales a estudiantes de pedagogía. Cada grupo estuvo compuesto por 10 sujetos. De este modo, la muestra quedó constituida por un $63 \%$ de personas de género femenino y $37 \%$ de género masculino. La edad de los sujetos fluctúa entre 19 y 27 años con una media de 20,3 años y desviación estándar de 2,5 años. La muestra total fue de 60 sujetos. Cabe señalar que los estudiantes participantes no han tenido un grado de contacto previo con respecto al concepto de autorregulación del aprendizaje.

\section{Procedimiento}

El procedimiento consistió en aplicar las entrevistas grupales en grupos de 10 estudiantes en una sala de clases. Antes de iniciar la entrevista grupal se indicó a los estudiantes que no se realizaría debate ni interacción entre ellos, sino que solamente se esperaba la respuesta de cada uno de forma independiente. De este modo, se realizaba la pregunta y cada estudiante respondía por turno a la pregunta. Cada entrevista grupal se grabó y luego se transcribió.

\section{Instrumentos y procedimiento de análisis de los datos}

Para recoger la información se generó un conjunto de preguntas que se aplicó en cada una de las entrevistas grupales de acuerdo con el procedimiento antes descrito. Las preguntas aplicadas a los estudiantes en las entrevistas grupales son:

1) ¿Qué comprenden por autorregulación del aprendizaje?

2) ¿Cómo planifican su estudio?

3) ¿Cómo estudian?

4) ¿Cómo verifican si están realizando bien y/o de forma adecuada su aprendizaje?

5) ¿Cómo identifican o precisan lo que deben aprender?

6) Si has obtenido malos resultados, ¿qué acciones toman para estudiar mejor?

7) ¿Qué dificultades tienen cuando se disponen a estudiar y durante su estudio?

Se realizaron 6 entrevistas grupales donde los participantes respondían a las preguntas sin interactuar entre ellos, ni generar debate. Las preguntas que se aplicaron en las entrevistas grupales se focalizaron en las siguientes dimensiones: 1) Concepto de autorregulación, 2) Fases del proceso de autorregulación del aprendizaje de acuerdo con el modelo de Zimmerman y 3) Dificultades en el proceso de autorregulación. La información recabada se transcribió y se procesó utilizando el software QDA Miner en su versión libre v1.4.5. El software se utilizó en etapas del análisis de contenido (Andréu, 2001).

Para el análisis de contenido se aplicó el modelo de desarrollo de categorías inductivas y deductivas según Mayring (2000). El modelo de categorías inductivas consiste en determinar las categorías a partir del texto obtenido en el proceso de investigación, es decir, de las respuestas a las preguntas. Este caso se aplicó para la categoría concepto de autorregulación del aprendizaje y para la categoría agentes anti regulación del aprendizaje. De este modo, la categoría concepto de autorregulación del aprendizaje se especificó desde los datos, dando origen a las siguientes subcategorías: Regular Emociones, Organización General previa a la planificación, Metacognición. Así mismo, para la categoría Agentes Anti regulación del Aprendizaje se 
especificó desde los datos las siguientes subcategorías: Desorganización y Distractores, Descontrol de Emociones, Desmotivación.

En el caso de la categoría Fases del Proceso de Autorregulación del Aprendizaje las subcategorías se determinaron usando el modelo deductivo. Para ello, se utilizó el modelo de Zimmerman desde el cual se emplean las siguientes subcategorías: Fase de Planificación, Fase de Ejecución, Fase de Auto Reflexión.

\section{RESULTADOS Y DISCUSIÓN}

La investigación se desarrolló bajo un paradigma interpretativo en la modalidad de investigación descriptiva. El análisis de datos se elabora en dos fases, la primera fase es cualitativa para la definición de categorías y subcategorías de análisis. La segunda fase es cuantitativa de asignación y recuento de categorías y subcategorías a través de frecuencias. El resultado del recuento de frecuencias por cada subcategoría se muestra en el gráfico de la Fig. 2 al finalizar la descripción de cada una de las categorías y subcategorías. Finalmente, la Fig. 3 representa una propuesta de mapa conceptual que muestra las relaciones entre categorías. Cabe señalar que en la categorización de las respuestas de los estudiantes se permitió la posibilidad de que un estudiante, ante la misma pregunta, incluyera información a recoger en dos subcategorías distintas. Es el caso, por ejemplo, de las respuestas a la pregunta ¿qué entiendes por autorregulación del aprendizaje?, debido a que estudiantes señalaban como respuesta la organización general previa al estudio lo que se puede etiquetar como concepto autorregulación del aprendizaje y como parte de la fase de planificación.

\section{Dimensión Autorregulación del Aprendizaje}

La dimensión autorregulación del aprendizaje permite describir las percepciones de los participantes en relación con tres categorías que son: Concepto Autorregulación del Aprendizaje, Fases del Proceso de Autorregulación del Aprendizaje y Agentes Anti Regulación del Aprendizaje. Cabe señalar que la categoría Agentes Anti Regulación del Aprendizaje, es una categoría que emergió de forma espontánea en el discurso de los participantes, incluso antes de llegar a consultar por las dificultades. A continuación, la Tabla 1 muestra la dimensión Autorregulación del Aprendizaje, sus categorías y subcategorías.

Tabla 1: Dimensión Autorregulación del Aprendizaje, categorías y subcategorías

\begin{tabular}{|c|c|l|}
\hline Dimensión & \multicolumn{1}{|c|}{ Categoría } & \multicolumn{1}{c|}{ Subcategoría } \\
\hline \multirow{4}{*}{$\begin{array}{c}\text { Autorregulación } \\
\text { del Aprendizaje }\end{array}$} & $\begin{array}{c}\text { Concepto Autorregulación } \\
\text { del Aprendizaje }\end{array}$ & $\begin{array}{l}\text { Regular Emociones } \\
\text { Organización General previa al estudio } \\
\text { Metacognición }\end{array}$ \\
\cline { 2 - 3 } & $\begin{array}{c}\text { Autorregulación } \\
\text { del Aprendizaje }\end{array}$ & $\begin{array}{l}\text { Fase de Planificación - Organización Inicial Básica } \\
\text { Fase de Ejecución - Auto Control Metacognitivo Base } \\
\text { Fase de Auto Reflexión }\end{array}$ \\
\cline { 2 - 3 } & $\begin{array}{c}\text { Agentes Anti Regulación } \\
\text { del Aprendizaje }\end{array}$ & $\begin{array}{l}\text { Desorganización y Distractores } \\
\text { Descontrol de Emociones } \\
\text { Desmotivación }\end{array}$ \\
\hline
\end{tabular}

Categoría Concepto Autorregulación del Aprendizaje: esta categoría hace referencia a la percepción de los estudiantes de pedagogía respecto al concepto de autorregulación del aprendizaje a través de la elaboración de una definición propia que explique el concepto. En este sentido, el concepto autorregulación del aprendizaje ha sido asociado con los siguientes conceptos que definen tres subcategorías que son: regular emociones, organización general previa a la planificación y metacognición. Las Tablas 2, 3 y 4 presentan algunos fragmentos de respuestas relacionadas a estas tres subcategorías.

Subcategoría Regular Emociones: Esta subcategoría señala que los estudiantes de pedagogía pueden definir la autorregulación del aprendizaje a través de la regulación de emociones. Algunos fragmentos de respuestas se presentan en la Tabla 2. Es importante señalar que esta definición obtuvo el segundo lugar de frecuencia en la categoría concepto de autorregulación. En este sentido, es relevante que los estudiantes de pedagogía tengan conciencia de la importancia del control de las emociones como lo han señalado Boekaerts y Corno (2005), y Kuhl (2000) entre otros, pues como futuros profesores deberán intervenir en aula ante situaciones de desregulación de emociones de sus estudiantes. 
Tabla 2: Fragmentos de respuestas de los participantes relacionados a la Subcategoría Regular Emociones

\begin{tabular}{|c|l|}
\hline Frecuencia & \multicolumn{1}{c|}{ Regular Emociones } \\
\hline \multirow{2}{*}{26} & $\begin{array}{l}\text { "...regular las emociones, regular mis emociones es para mí autorregular mi aprendizaje es como } \\
\text { una condicionante para que yo pueda regular mi aprendizaje..." } \\
\text { "...y debo regular mis emociones para autorregular mi aprendizaje, el problema es que no sé } \\
\text { cómo..." }\end{array}$ \\
\hline
\end{tabular}

Subcategoría Organización General previa al estudio: La subcategoría organización general previa al estudio indica que los estudiantes de pedagogía pueden definir la autorregulación del aprendizaje a través de la concepción de la organización general que realizan de forma previa al estudio. Por ello, se advierte que no es una planificación específica, ni estratégica para el desarrollo de una actividad académica. Así, ellos definen una forma general de ordenarse. Esta subcategoría obtuvo el primer lugar de frecuencia en la categoría concepto de autorregulación del aprendizaje, lo que nos sugiere que los participantes comprenden, en su mayoría, que este tipo de organización general podría definir a la autorregulación del aprendizaje. En la Tabla 3 se muestran algunos fragmentos representativos.

Tabla 3: Fragmentos de respuestas de los participantes relacionados a la Subcategoría Organización previa a la planificación

\begin{tabular}{|c|l|}
\hline Frecuencia & \multicolumn{1}{c|}{ Organización general previa al estudio } \\
\hline 28 & $\begin{array}{l}\text { “... autorregulación del aprendizaje es como yo me organizo. Yo hago una lista de las cosas que } \\
\text { tengo que hacer al día. Si tengo que estudiar... cuanto dedico a esta u otra cosa, esa es mi forma } \\
\text { de autorregularme en el aprendizaje..." }\end{array}$ \\
\hline
\end{tabular}

Subcategoría Metacognición: Esta subcategoría señala la posibilidad de definición de autorregulación del aprendizaje usando el concepto de metacognición que se refiere al proceso de auto reflexión que realizan los sujetos al juzgar su actuar y reaccionar ante su propio auto juicio (Zimmerman y Moylan, 2009). En esta investigación sólo dos estudiantes de pedagogía indicaron como posible definición de autorregulación del aprendizaje a la metacognición. En la Tabla 4 se presentan fragmentos de respuestas de los participantes relacionados a esta subcategoría.

Tabla 4: Fragmentos de respuestas de los participantes relacionados a la subcategoría Metacognición

\begin{tabular}{|c|l|}
\hline Frecuencia & \multicolumn{1}{c|}{ Metacognición } \\
\hline \multirow{6}{*}{6} & $\begin{array}{l}\text { "...autorregulación del aprendizaje es para mí la auto evaluación y reflexión que yo hago de mis } \\
\text { resultados académicos una vez que tengo las notas y al final del semestre para pensar en que } \\
\text { "...en mi caso yo creo que autorregulación del aprendizaje es cuando yo me auto evaluó y } \\
\text { reviso que hice mal y que hice bien, para evitar caer en el mismo error y que eso me perjudiqué } \\
\text { en mi rendimiento y mis notas..." }\end{array}$ \\
\hline
\end{tabular}

Categoría Fases del Proceso de Autorregulación del Aprendizaje: esta categoría hace mención de la percepción de los estudiantes de pedagogía respecto al proceso de autorregulación del aprendizaje. Para realizar el análisis se hará referencia al modelo cíclico de fases de autorregulación del aprendizaje de Zimmerman y Moylan (2009). Estos autores conciben la autorregulación del aprendizaje como un proceso cíclico de tres fases: fase de planificación, fase de ejecución y fase de auto reflexión. A continuación, las Tablas 5,6 y 7 presentan algunos fragmentos de respuestas relacionadas a cada fase del proceso de autorregulación.

Sub Categoría Fase de Planificación: Los procesos de la fase de planificación son: análisis de la tarea y creencias auto-motivadoras. En relación con el primer proceso se muestra que los estudiantes declaran realizar una planificación orientada a la meta de obtener una nota para aprobar un curso. Esto coincide con lo planteado por Panadero y Alonso-Tapia (2014) quienes señalan que en esta etapa el estudiante "analiza la tarea, valora su capacidad para realizarla con éxito, establece sus metas y planifica" (Panadero y AlonsoTapia, 2014, p.451). No obstante, se advierte que los estudiantes no planifican de forma estratégica pensando en su aprendizaje, sino que lo hacen de forma general y pensando en obtener una nota que les permita aprobar sus asignaturas, este resultado es similar a la investigación de Zambrano (2016). Lo anterior se muestra en la Tabla 5 que presenta fragmentos representativos de respuestas de los participantes en relación con la fase de planificación. 
Cabe señalar que la planificación es un predictor del éxito que se tendrá en la tarea, así a mayor tiempo de planificación mejores resultados se obtendrán (Zimmerman, 2008). En efecto, varias investigaciones señalan que la mayor diferencia entre aprendices expertos y novatos se debe al tiempo que dedican a la planificación (Ericsson, Charness, Feltovich y Hoffman, 2006; Zimmerman y Kitsantas, 2005). En el caso de los estudiantes que participan en este estudio no existe conciencia de que una buena planificación estratégica tanto a corto plazo como largo plazo que sea orientada al aprendizaje los puede beneficiar en el contexto de las habilidades del siglo XXI específicamente en la habilidad de aprender a aprender (Trilling y Fadel, 2009). En suma, se advierte que no existe planificación estratégica, sino que una organización básica previa al estudio.

Tabla 5: Fragmentos de respuestas de los participantes relacionados a la Subcategoría Fase de Planificación

\begin{tabular}{|c|l|}
\hline Frecuencia & \multicolumn{1}{c|}{ Fase de Planificación - Organización Inicial Básica } \\
\hline 39 & $\begin{array}{l}\text { "...yo concuerdo planifico y establezco mi meta asociada a la nota porque tengo que aprobar mis } \\
\text { ramos..." } \\
\text { “...la planificación que realizo está pensada en lograr la nota que necesito para aprobar y obtener los } \\
\text { beneficios de becas que tengo y las necesito..." }\end{array}$ \\
\hline
\end{tabular}

Desde la perspectiva de las creencias auto-motivadoras se advierte que los estudiantes no son conscientes de la importancia de las variables que generan y sostienen su motivación para realizar una actividad, tal como autoeficacia y expectativas, entre otras. Esto se debe a que en sus respuestas está ausente la declaración o descripción de auto motivación por el aprendizaje. No obstante, su motivación es extrínseca y orientada a la meta que se manifiesta al declarar que desarrollan sus actividades académicas para obtener una nota que les permite aprobar sus asignaturas. Es importante indicar que existe evidencia empírica que señala que los estudiantes con metas de aprendizaje escogen y utilizan estrategias que los dirigen a aprendizajes profundos, tienen procesos de reflexión, se recuperan antes de fracasos académicos y su motivación es intrínseca (Grant y Dweck, 2003; Harackiewicz, Barron y Elliot, 1998). Por ello es importante que los estudiantes trabajen en menor grado por una nota y se centren mayormente en su proceso de aprendizaje.

Sub Categoría Fase de Ejecución: En la fase de ejecución se distinguen dos procesos que son: auto observación y auto control como señalan Zimmerman y Moylan (2009). En este sentido, los estudiantes declaran utilizar procesos de autocontrol de tipo metacognitivo al señalar que utilizan estrategias específicas tales como subrayar un texto o hacer un resumen al estudiar. No obstante, no declaran realizar de forma efectiva el proceso de autocontrol motivacional. Por ello, aparecen recurrentemente respuestas que tienen que ver con obstáculos para la autorregulación del aprendizaje que se relacionan con la desorganización, distractores, descontrol de emociones y desmotivación. Tampoco declaran realizar el proceso de auto observación que implica una comparación entre lo que se está desarrollando con el modelo ideal de ejecución.

En este sentido, es frecuente que el estudiante no realice el proceso de auto observación durante la ejecución de una actividad lo que podría deberse a los siguientes motivos: i) en muchas ocasiones el estudiante no tiene o no sabe escoger un modelo a seguir para comparar su desarrollo, ii) el estudiante no es consciente de la importancia que tiene revisar su proceso de aprendizaje iii) la evaluación que realiza el profesor es al producto final, de este modo, el proceso queda en un segundo plano u olvidado. En suma, los estudiantes participantes del estudio no realizan un proceso de monitoreo durante la ejecución de su estudio. La Tabla 6 muestra fragmentos representativos de respuestas de los participantes relacionados a la fase de ejecución asociada a los procesos de autocontrol de tipo metacognitivo, caso en el que el resumen aparece como la técnica de estudio pues les permite memorizar lo que es más importante para una prueba.

Tabla 6: Fragmentos de respuestas de los participantes relacionados a la Subcategoría Fase de Ejecución

\begin{tabular}{|c|l|}
\hline Frecuencia & \multicolumn{1}{c|}{ Fase de Ejecución - Auto Control Metacognitivo Base } \\
\hline \multirow{2}{*}{22} & $\begin{array}{l}\text { "...Pero con los ramos en los que me va bien es pura memorización y lo que en ellos tengo } \\
\text { que aplicar es sólo eso. Por el mismo tema de la estrategia que ejecuto que es repetir y así } \\
\text { memorizar, pero nunca he sabido si he aprendido con esos ramos..." } \\
\text { "...Para mí lo que funciona son los resúmenes como había dicho antes..." } \\
\text { "...Mis amigas me prestan sus resúmenes...o los hacemos juntas en la biblioteca central...pero } \\
\text { igual que el resto debo reconocer que no conozco mi técnica de estudio" “...La que podría } \\
\text { indicar que ocupo es el resumen cuando estudio..." } \\
\text { "...Yo estudio subrayando...leo y subrayo lo que me parece importante..." } \\
\text { "....siempre nos preocupamos del resultado final, de la nota y así los resúmenes ayudan mucho, } \\
\text { es la síntesis de lo que tengo que memorizar..." }\end{array}$ \\
\hline
\end{tabular}


Sub Categoría Fase de Auto Reflexión: De acuerdo con el modelo de Zimmerman y Moylan (2009), la fase de auto reflexión se compone del proceso de auto juicio y de la auto reacción. En este sentido, los resultados relacionados a esta fase muestran que los estudiantes reflexionan sobre su proceso de aprendizaje realizando auto juicios al final del proceso cuando ya tienen la nota y pueden revisar el certamen. Los auto juicios son los procesos por los cuales el estudiante juzga su ejecución y que influyen en su auto reacción (Panadero y Alonso-Tapia, 2014). La Tabla 7 muestra fragmentos de respuestas representativas relacionados a la fase de auto reflexión.

Tabla 7: Fragmentos de respuestas de los participantes relacionados a la Subcategoría Fase de Auto Reflexión

\begin{tabular}{|c|l|}
\hline Frecuencia & \multicolumn{1}{c|}{ Fase de Auto Reflexión } \\
\hline \multirow{1}{*}{17} & $\begin{array}{l}\text { "...La forma en que yo mido si estoy aprendiendo o no, es como poniéndome a prueba si después } \\
\text { re un tiempo me acuerdo de lo que estuve aprendiendo, es curioso porque me pasa en muchos } \\
\text { memorizado más para la prueba..." }\end{array}$ \\
$\begin{array}{l}\text { "...Analizar los resultados, los factores. Si no estudie o estudie tarde no hay nada que analizar. } \\
\text { Aprovechar las horas que dan los profes para revisar los certámenes y preguntarle directamente } \\
\text { en que me fue mal y así poder corregir los errores..." } \\
\text { "...Ver en que falle, revisar el certamen, anoto en lo que falle y todo y para revisarlo, para volver } \\
\text { a entenderlo y cosas así, estudiar más para el próximo certamen..." }\end{array}$ \\
\hline
\end{tabular}

Categoría Agentes Anti Regulación del Aprendizaje: esta categoría hace referencia a la percepción de los estudiantes de pedagogía respecto a los agentes que dificultan u obstaculizan la regulación de su aprendizaje. En este contexto, los agentes anti regulación han sido asociados con las siguientes subcategorías: Desorganización y Distractores, Descontrol de Emociones y Desmotivación. Se creo esta categoría debido a la recurrente descripción por parte de los estudiantes de este tipo de agentes anti regulación del aprendizaje. Lo anterior, está en concordancia, con lo señalado en la categoría Fase de Ejecución donde se evidencio una carencia del proceso de autocontrol motivacional.

Sub Categoría Desorganización y Distractores: Los resultados asociados a esta subcategoría se relacionan con las dificultades que señalan tener los estudiantes de pedagogía respecto de su propia organización y que influyen de forma negativa en la regulación de su aprendizaje. La Tabla 8 muestra fragmentos representativos de respuestas de los participantes relacionados a la desorganización y distractores, los resultados coinciden con los presentados en Zambrano (2016).

Tabla 8: Fragmentos de respuestas de los participantes relacionados a la Subcategoría Desorganización y Distractores

\begin{tabular}{|c|c|}
\hline Frecuencia & \multicolumn{1}{c|}{ Desorganización y Distractores } \\
\hline 19 & $\begin{array}{l}\text { "...Cuando estudio en la casa...pierdo mucho tiempo, me dan ganas de hacer cualquier } \\
\text { cosa, así estoy el último día estudiando ...soy un desorden..." } \\
\text { "...Yo quiero comentar lo que pasa para los certámenes...le dedicamos la noche anterior, } \\
\text { "centralazo con café toda la noche", como mucha cantidad y calidad no suele estar tan mala } \\
\text { cuando es tanta la presión. Pero la calidad de vida es la que baja..." }\end{array}$ \\
\hline
\end{tabular}

Sub Categoría Descontrol de Emociones: En esta subcategoría se asocian las respuestas referidas a la dificultad para controlar emociones. El control de las emociones es fundamental para la regulación del aprendizaje pues en los casos donde no se controlan existirá interferencia con su aprendizaje como lo señalan Boekaerts y Corno (2005). La Tabla 9 muestra fragmentos representativos de las respuestas de los participantes relacionados al descontrol de emociones.

Tabla 9: Fragmentos de respuestas de los participantes relacionados a la Subcategoría Descontrol de Emociones

\begin{tabular}{|c|l|}
\hline Frecuencia & \multicolumn{1}{c|}{ Descontrol de Emociones } \\
\hline \multirow{2}{*}{23} & $\begin{array}{l}\text { “...Muchas veces me encanta el ramo, entiendo todo, pero llega el certamen y me va muy } \\
\text { mal creo que no controlo la ansiedad se me olvida todo. Pero luego me doy cuenta de que se } \\
\text { todo y no entiendo porque no me fue tan bien y quizás es porque a veces inciden más cosas } \\
\text { en los certámenes como la concentración y las emociones..." } \\
\text { "...Yo quiero agregar algo que son los hechos inesperados, por ejemplo, yo me regulo, yo } \\
\text { quiero dejar de estar estresado, pero llega una situación que altera todo ese ritmo y } \\
\text { descontrola mis emociones... Y concuerdo en que debo regular mis emociones, pero no sé } \\
\text { cómo..." }\end{array}$ \\
\hline
\end{tabular}


Sub Categoría Desmotivación: La sub categoría desmotivación representa los resultados asociados a los problemas de desmotivación descritos por los participantes del estudio. La motivación juega un rol importante en la autorregulación del aprendizaje debido a que representa el motor que moviliza al estudiante para realizar acciones y tomar decisiones que le permitan lograr sus metas u objetivos. En este sentido, el control de la motivación ha sido estudiado por Kuhl $(1994,2000)$ quien enfatiza en el rol de las emociones y cómo estas pueden obstaculizar el inicio de las tareas u actividades que el estudiante debe realizar. En la Tabla 10 se muestran fragmentos representativos de las respuestas relacionados con la desmotivación y que coinciden con lo planteado por Kuhl (2000).

Tabla 10: Fragmentos de respuestas de los participantes relacionados a la Desmotivación

\begin{tabular}{|c|l|}
\hline Frecuencia & \multicolumn{1}{c|}{ Desmotivación } \\
\hline & $\begin{array}{l}\text { “... yo me desmotivo porque muchos ramos son desmotivantes. No tienen sentido de } \\
\text { utilidad que es lo que le da sentido a una asignatura, cuando se establece para que te sirve } \\
\text { algo y por qué está en tu formación. Cuando no sabes para que te sirve algo puedes perder } \\
\text { el interés en la clase y eso mismo hará que te desconcentres...” } \\
\text { "...escuchar la clase sin hacer nada más es desmotivante.... }\end{array}$ \\
\hline
\end{tabular}

La Tabla 11 muestra el resumen de sub categorías y frecuencias donde se observa que las frecuencias son bajas en relación con el total de participantes que son 60 sujetos. Luego, la Fig. 2 presenta un gráfico de frecuencias totales por cada subcategoría con el objetivo de resumir el análisis cualitativo antes descrito. Así, en la Fig. 2 se observa que la subcategoría metacognición tiene la frecuencia más baja y que la subcategoría organización general previa al estudio presenta la frecuencia más alta. Ambas subcategorías pertenecen a la categoría concepto autorregulación del aprendizaje y los resultados obtenidos nos señalan que los estudiantes de pedagogía comprenden, en primera instancia en su mayoría, que la organización general que realizan representa al concepto de autorregulación del aprendizaje. No obstante, cabe señalar que las tres subcategorías del Concepto Autorregulación del Aprendizaje son parte de la autorregulación del aprendizaje, pero los estudiantes no indicaron a las tres en conjunto como concepto, sino que lo realizaron por separado. Respecto a las fases del proceso de autorregulación del aprendizaje, en la Fig. 2 se observa que tanto la fase de planificación como la fase de ejecución presentan las frecuencias mayores. Esto ocurre porque los estudiantes de pedagogía señalaron en su mayoría realizar una planificación general y utilizar técnicas como el subrayado y resúmenes durante la ejecución de una actividad académica. No obstante, en ambas fases el desarrollo que declaran es incipiente en relación con: 1) carencia de planificación estratégica en la fase de planificación, 2) falta de procesos de autocontrol motivacional lo que influye en que aparecen en su discurso los agentes anti regulación del aprendizaje tales como: distractores y descontrol de emociones, 3) ausencia de auto-registros que les permitan comparar y monitorear la ejecución de su aprendizaje.

En suma, los mayores agentes anti regulación del aprendizaje son el descontrol de emociones y la desorganización, seguido por la desmotivación, lo que coincide con el planteamiento de Kuhl $(1994,2000)$. Kuhl señala que los estudiantes que no regulan sus emociones pueden orientarse al estado y no a la acción. De este modo, se mantienen en estados de preocupación u otros estados emocionales que no les permiten iniciar, avanzar o ejecutar sus actividades académicas. Es importante señalar que definir el concepto de autorregulación del aprendizaje es complejo por la naturaleza multidimensional de su constructo, y a pesar de que existen varios modelos que ayudan a comprender el concepto de autorregulación del aprendizaje, ninguno de ellos lo explica completamente (Panadero y Alonso-Tapia, 2014). Por ello, en esta investigación se ha utilizado dos modelos, el de Zimmerman y el de Kuhl para apoyar la comprensión del fenómeno en estudio.

Tabla 11: Resumen de Sub Categorías y frecuencias

\begin{tabular}{|c|c|}
\hline Sub Categorías & Frecuencia \\
\hline \multicolumn{2}{|c|}{ Sub Categorías de la Categoría Concepto Autorregulación del Aprendizaje } \\
\hline Regular Emociones & 26 \\
\hline Auto Evaluación & 6 \\
\hline Organización General Previa al Estudio & 28 \\
\hline \multicolumn{2}{|c|}{ Sub Categorías de la Categoría Fases de Proceso de Autorregulación del Aprendizaje } \\
\hline Fase de Planificación - Organización Inicial Básica & 39 \\
\hline Fase de Ejecución - Auto Control Metacognitivo Base & 22 \\
\hline Fase de Auto Reflexión & 17 \\
\hline
\end{tabular}


Tabla 11: (continuación).

\begin{tabular}{|l|c|}
\hline Sub Categorías de la Categoría Agentes Anti regulación del Aprendizaje \\
\hline Desorganización y Distractores & 19 \\
\hline Descontrol Emociones & 23 \\
\hline Desmotivación & 28 \\
\hline
\end{tabular}

SubCateg. Agentes Antiregulacion del Aprendizaje

Desmotivacion

DescontrolEmociones

DesorganizacionyDistractores

SubCateg. Fases de Proceso de Autorregulación del Aprendizaje

Fase de Auto Reflexion

Fase de Ejecución - Auto Control Metacognitivo Base

Fase de Planificacion - Organización Inicial Básica

SubCateg. Concepto Autorregulación del Aprendizaje

Organización General Previa al Estudio

Metacognición

RegularEmociones
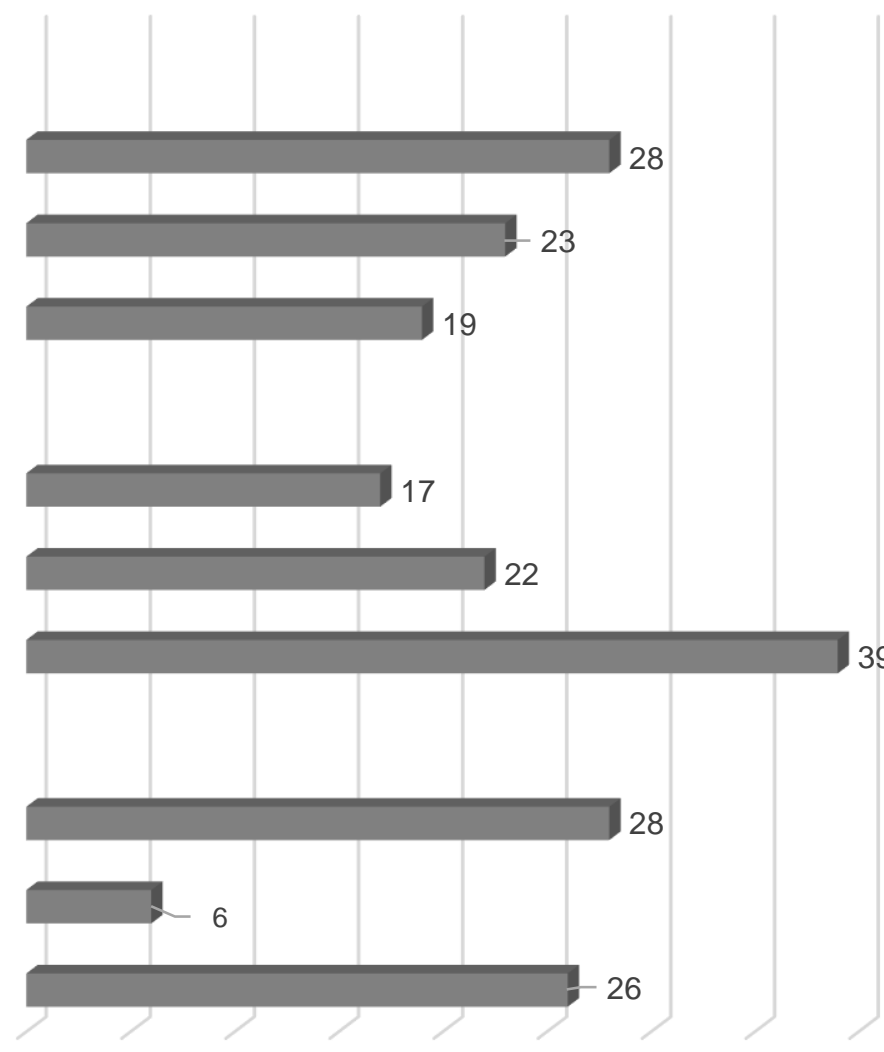

0

5

10

15

20

25

30

35

40

Fig. 2: Gráfico de frecuencias totales por subcategoría

La Fig. 3 presenta una propuesta de mapa conceptual que muestra las relaciones entre las categorías usadas en el estudio. Se observa que la categoría agentes anti-regulación del aprendizaje se relaciona con la categoría fases del proceso de autorregulación del aprendizaje a través de la relación de obstaculización. Además, la categoría concepto autorregulación del aprendizaje se relaciona con la categoría fases del proceso de autorregulación del aprendizaje por medio de la compresión, esto significa que no es posible aplicar de forma adecuada las fases del proceso de autorregulación sino se tiene conciencia y comprensión del concepto de autorregulación del aprendizaje. Por lo anterior, es necesario enseñar a los estudiantes cómo se aprende a través del conocimiento de los modelos que explican cómo se debería autorregular el aprendizaje de tal forma que ellos sean conscientes de lo que significa aprender, que falencias tienen y como podrían mejorar. De esta forma se fomenta esta habilidad que requerirán por el resto de su vida en el contexto actual de sociedad (Trilling y Fadel, 2009).

Cabe señalar que en la Fig. 3 la categoría Concepto Autorregulación del aprendizaje muestra tres subcategorías que son Regular Emociones, Organización general previa al estudio y Meta Cognición. No obstante, a pesar de que la relación que las une al concepto se denomina "es parte de" ningún estudiante señalo una descripción usando las tres componentes para referirse al concepto de autorregulación del aprendizaje. Luego, la relación entre el Concepto Autorregulación del aprendizaje y las Fases del Proceso de Autorregulación del Aprendizaje es "se requiere comprender" lo que implica como se mencionó anteriormente que para profundizar en el detalle de cada una de las fases del proceso de autorregulación primero es necesario tener una noción clara de lo que significa el concepto.

En el caso de las Fases del Proceso de Autorregulación del Aprendizaje en la Figura 3 se observa que está compuesta por Fase de Planificación con Organización Inicial Básica, Fase de Ejecución con Auto Control 
Metacognitivo Base y Fase de Reflexión. En este sentido, es importante señalar que existe diferencia con respecto al modelo de Zimmerman pues los estudiantes no declaran, ni describen realizar procesos como el autocontrol motivacional de la fase de ejecución y además el autocontrol metacognitivo es básico por ello se denominó "Auto Control Metacognitivo Base".

Luego, la categoría Agentes anti-regulación del aprendizaje se relaciona con las Fases del Proceso de Autorregulación del Aprendizaje a través de la relación "obstaculizan" porque la Desorganización y distractores, Descontrol de Emociones y Desmotivación son impedimentos que pueden obstruir el proceso de aprendizaje del estudiante en cualquier fase del proceso y no sólo en la fase de ejecución.

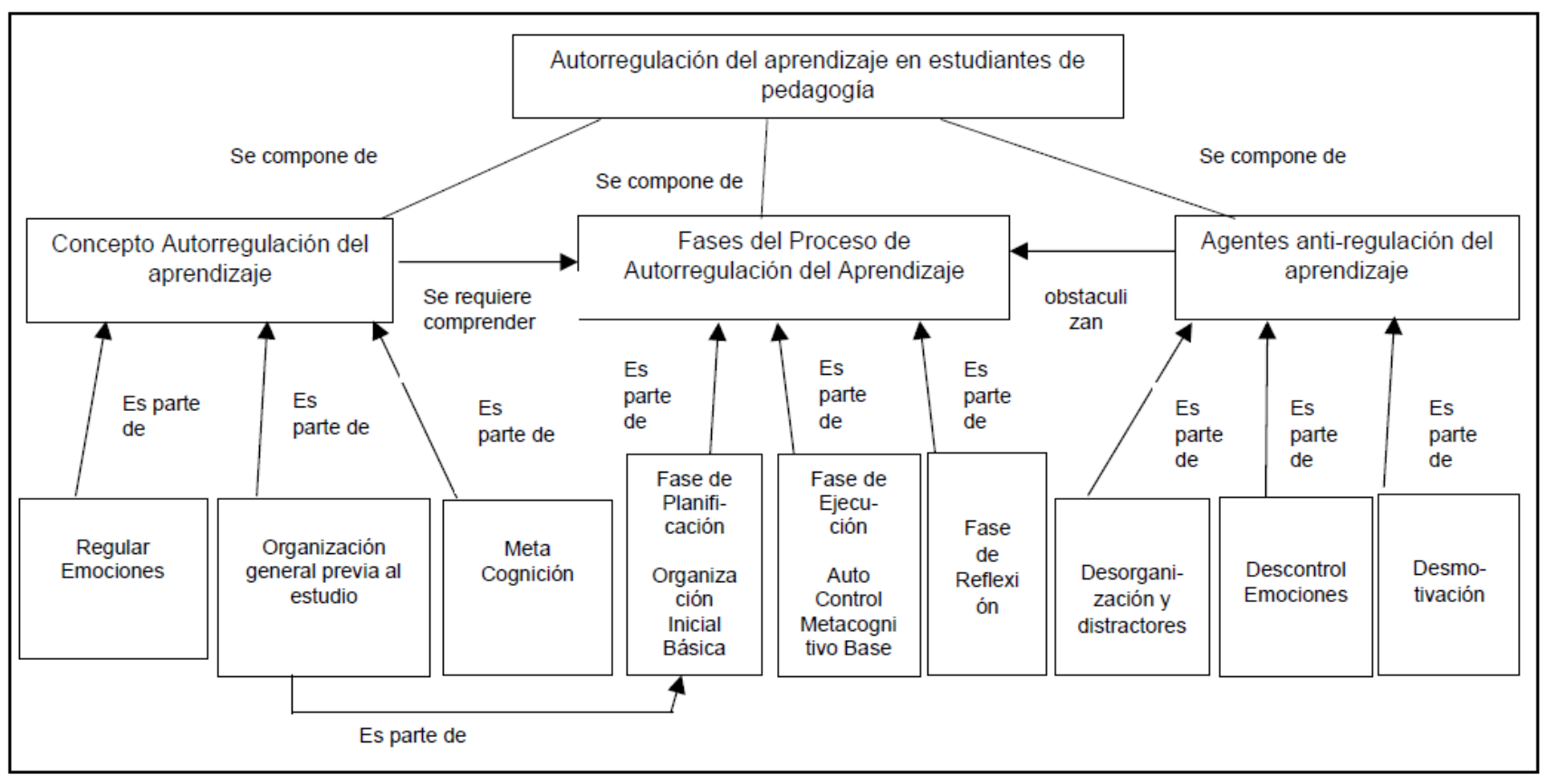

Fig. 3: Propuesta de modelo conceptual que muestra las relaciones entre categorías del estudio

En suma, cabe señalar que es necesario que el estudiante comprenda el concepto de autorregulación del aprendizaje para que pueda a través de los enfoques que proponen los modelos, ser consciente de los procesos y estrategias que puede llevar a cabo para mejorar su propio aprendizaje. De esta forma, además de mejorar la eficacia del estudio, también se podría mejorar el uso eficiente del tiempo, con tal de que para el estudiante sea significativo el proceso metacognitivo de la autorregulación, evitando las prácticas y estrategias de estudio que conllevan a considerar el aprendizaje como el resultado de la acción reproductiva de los saberes y contenidos de las asignaturas, propiciando, a través de la concientización, un proceso reflexivo sobre lo que se aprende y cómo se aprende.

En este sentido, coincidimos con Hernández y Camargo (2017) que señalan: "la labor de caracterizar al estudiante universitario iberoamericano resulta imprescindible en procura de identificar sus dimensiones como un sujeto autorregulador de su proceso de aprendizaje. Esta información es de central importancia al momento de diseñar e implementar planes, programas y acciones en los escenarios formativos que contribuyan al avance de los estudiantes en sus estudios académicos, así como la promoción de pautas para la generación de autonomía estudiantil entendida como autorregulación del aprendizaje" (Hernández y Camargo, 2017).

Finalmente, se debe mencionar que el estudio cuenta con limitaciones tales como, el tamaño de la muestra que consideró a 60 sujetos y también el hecho de que se realizó el estudio sólo bajo el paradigma cualitativo. No obstante, estas limitaciones pueden ser remediadas en futuras investigaciones que se llevan a cabo usando instrumentos de autoinforme con muestras superiores a 300 estudiantes. Y estudios cualitativos con muestras de estudiantes de otras áreas (como estudiantes del área salud). Porque se puede utilizar la misma pauta de preguntas de este estudio, que ha tenido el propósito de ser aplicada como un piloto para dirigir las siguientes investigaciones que permitirán realizar procesos de diagnóstico en estudiantes de primer año. También, es interesante referir que existen investigaciones en estudiantes universitarios del área ingeniería que han evidenciado resultados equivalentes (Zambrano, 2016) en relación con la carencia de planificación y monitoreo de su proceso de aprendizaje. 


\section{CONCLUSIONES}

Las conclusiones de este estudio son:

1) En relación con la comprensión que tienen los estudiantes participantes sobre el concepto de autorregulación del aprendizaje, se advierte que se refieren principalmente a la organización general previa al estudio y a la regulación de emociones. En este sentido, cabe señalar que, la organización que realizan no está orientada al propósito de su aprendizaje, sino que está orientada a obtener una nota.

2) Respecto al proceso de autorregulación del aprendizaje se concluye que los estudiantes no realizan una planificación estratégica y que, al ejecutar una actividad académica, utilizan mayormente los resúmenes. No obstante, se sugiere que no han desarrollado cada una de las fases de forma exhaustiva, ya que, por ejemplo, la fase de planificación no es estratégica. Adicionalmente, en la fase de ejecución no es común que realicen auto registros que les permitan comparar las acciones previas con la nueva ejecución.

3) En lo referente a las dificultades para regular su aprendizaje, se obtuvo que las emociones no controladas, tales como, la desorganización y las distracciones, constituyen impedimentos que obstaculizan el aprendizaje. Sin embargo, se debe destacar que los estudiantes son conscientes y reflexionan sobre estos obstáculos.

4) En síntesis, la fase de planificación y ejecución son incipientes en relación con: i) carencia de planificación estratégica en la fase de planificación, ii) falta de procesos de autocontrol motivacional lo que influye en que aparecen en su discurso descripciones de agentes anti regulación del aprendizaje tales como: distractores y descontrol de emociones, iii) ausencia de auto-registros que les permitan comparar y monitorear la ejecución de su aprendizaje. Por ello, los estudiantes participantes del estudio no describen realizar un proceso de monitoreo durante la ejecución de su estudio.

\section{AGRADECIMIENTOS}

Esta investigación se realizó durante el proceso de definición del proyecto de tesis doctoral de la autora Carolina Zambrano Matamala para optar a la candidatura al grado de doctor en Educación de la Universidad de Concepción, Chile. Se agradece a los revisores del artículo por sus aportes a esta investigación.

\section{REFERENCIAS}

Andréu, J., Las técnicas de análisis de contenido: Una revisión actualizada. Documento de trabajo, S2001/03, Centro de estudios andaluces, 2001, https://goo.gl/3tFUFG, Acceso: 10 de junio (2017)

Boekaerts, M. y Niemivirta, M., Handbook of self-regulation, 1를., 417-451, Elsevier Academic Press, San Diego, CA (2000)

Boekaerts, M., Motivated learning: Studying student situation transactional units, doi:10.1007/bf03173110, European Journal of Psychology of Education, 14(1), 41-55 (1999)

Boekaerts, M., Pintrich, P.R., y Zeidner, M., Handbook of self-regulation, 2ª Ed., 417-451, Elsevier Academic Press, San Diego, CA (2005)

Boekaerts, M., y Corno, L., Self-regulation in the classroom: A perspective on assessment and intervention, doi:10.1111/j.1464-0597.2005.00205.x, Applied Psychology, 54(2), 199-231 (2005)

Chaves, E., Trujillo, J.M., y López, J.A., Autorregulación del aprendizaje en entornos personales de aprendizaje en el Grado de Educación Primaria de la Universidad de Granada, España, doi:10.4067/S071850062015000400008, Formación Universitaria, 8(4), 63-76 (2015)

Cuba-Esquivel, A., Constructo competencia: síntesis histórico-epistemológica, doi:10.18800/educacion.201601.001, Educación, 25(48), 7-27 (2016)

Daura, F., Aprendizaje autorregulado y rendimiento académico en estudiantes del ciclo clínico de la carrera de Medicina, Revista Electrónica de Investigación Educativa, ISSN: 1607- 4041, 17(3), 13-27, 2015, goo.gl/cCz411. Acceso: 10 de mayo (2017)

Díaz, A., Pérez, M.V., Valenzuela, M., Muñoz, P., Rivas, S., y Salas, C., Procesos de autorregulación del aprendizaje en estudiantes universitarios de primer año, doi: 10.17060/ijodaep, Internacional Journal of Developmental an Educational Psychology, 4(1), 789-800 (2010)

Donoso, S., Donoso, G., y Ó. Arias, Iniciativas de retención de estudiantes de educación superior, Revista Calidad en la Educación, ISSN: 0718-4565, 33(2), 15-61, 2010, goo.gl/A1f4aB. Acceso: 5 de junio (2017)

Ericsson, K. A., Charness, N., Feltovich, P.J., y Hoffman, R. The Cambridge handbook of expertise and expert performance, 2 ${ }^{a}$ Ed., 150-198. Cambridge: Cambridge University Press, Londres, Inglaterra (2006)

Garello, M. V., y Rinaudo, M. C., Autorregulación del aprendizaje, feedback y transferencia de conocimiento: Investigación de diseño con estudiantes universitarios, Revista electrónica de investigación educativa, ISSN: 1607- 4041, 15(2), 131147, 2013. Acceso: 05 de mayo (2017) 
Grant, H., y Dweck, C. S., Clarifying achievement goals and their impact, doi: 10.1037/0022-3514.85.3.541, Journal of Personality and Social Psychology, 85(3), 541-553 (2003)

Harackiewicz, J.M., Barron, K. E., y Elliot, A. J., Rethinking achievement goals: When are they adaptive for college students and why?, doi:10.1207/s15326985ep3301_1, Educational Psychologist, 33(1), 1-21 (1998)

Hernández, A. y Camargo, Á., Autorregulación del aprendizaje en la educación superior en lberoamérica: una revisión sistemática, doi:10.1016/j.rlp.2017.01.001, Revista Latinoamericana de Psicología, 49(2), 146-160 (2017)

Hernández, R., Fernández, C., y Baptista, P., Metodología de la Investigación, 5a Ed., 200-251, McGraw-Hill, México (2010) Kuhl, J., Handbook of Self-Regulation,1 ${ }^{a}$ Ed., 111-169, Academic Press, San Diego, CA (2000)

Kuhl, J., Volition and personality: Action versus state orientation, 1르. Ed., 9-46, Hogrefe y Huber, Seattle (1994)

Mayring, P., Qualitative Content Analysis, doi: 10.17169/fqs-1.2.1089, Forum Qualitative Sozialforschung / Forum: Qualitative Social Research, 1(2), Art. 20 (2000)

Panadero, E., A review of self-regulated learning: Six models and four directions for research, doi:10.3389/fpsyg.2017.00422, Frontiers in Psychology, 8(422), (2017)

Panadero, E., y Alonso -Tapia, J., ¿Cómo autorregulan nuestros estudiantes? Revisión del modelo cíclico de Zimmerman sobre autorregulación del aprendizaje, doi:10.6018/analesps.30.2.167221, Anales de Psicología, 30(2), (2014)

Pintrich, P., Handbook of Self-Regulation, 1르 Ed., 452-502, Academic Press, San Diego, CA (2000)

Pintrich, P., y De Groot, E.V., Motivational and Self-Regulated Learning Components of Classroom Academic Performance, doi:10.1.1.315.6253, Journal of Educational Psychology, 82(1), 33-40 (1990)

Pool-Cibrián, W., y Martínez-Guerrero, J., Autoeficacia y uso de estrategias para el aprendizaje autorregulado en estudiantes universitarios, goo.gl/DVuDRU, ISSN: 1607- 4041, Revista Electrónica de Investigación Educativa, 15(3), 21 37 (2013)

Pranke, A., y Bragagnolo Frison, L. M., Potencialização da Aprendizagem Autorregulada de Bolsistas do PIBID/UFP el do curso de Licenciatura em Matemática através de Oficinas Pedagógicas, doi:10.1590/1980-4415v29n51a12, Boletim de Educação Matemática, 29(51), (2015)

Rosário, P., Pereira, A., Hõgemann, J., Nunes, A. R., Figueiredo, M., Núñez, J. C., y Gaeta, M. L., Autorregulación del aprendizaje: una revisión sistemática en revistas de la base SciELO, doi: 10.11144/Javeriana.UPSY13-2.aars, Universitas Psychologica, 13(2), 781-797 (2014)

Simão, A.M.V. y Flores M.A., Student-centred methods in higher education: Implications for student learning and professional development, The International Journal of Learning, ISSN: 1447-9494, 17(2), 207-218, 2010, goo.gl/umpbns, Acceso: 8 de abril (2017)

Sitzmann, T., y Ely, K. A meta-analysis of self-regulated learning in work-related training and educational attainment: What we know and where we need to go, doi: 10.1037/a0022777, Psychological Bulletin, 137(3), 421-442, (2011)

Trilling, B. y Fadel, C., 21st century skills - learning for Life in Our Times, $1^{a}$ Ed., 45-60, John Wiley \& Sons, San Francisco, CA (2009)

Winne, P. H., A metacognitive view of individual differences in self-regulated learning, doi:10.1016/S1041-6080(96)900229, Learning and Individual Differences, 8(4), 327-353 (1996)

Winne, P. H., Experimenting to bootstrap self-regulated learning, doi: 10.1037/0022-0663.89.3.397, Journal of Educational Psychology, 89(3), 397-410 (1997)

Zambrano, C., Autoeficacia, Prácticas de Aprendizaje Autorregulado y Docencia para fomentar el Aprendizaje Autorregulado en un Curso de Ingeniería de Software, doi:10.4067/S0718-50062016000300007, Formación Universitaria, 9(3), 51-60 (2016)

Zimmerman, B. J., Handbook of self-regulation, 1르 Ed., 13-40, Academic Press, San Diego, California (2000)

Zimmerman, B. J., Motivation and self-regulated learning. Theory, research and applications, $1^{\text {a }}$ Ed., 267-295, Lawrence Erlbaum Associates, New York (2008)

Zimmerman, B. J., y Campillo, M., The Psychology of problem solving, 1를., 233-262, Cambridge University Press, New York (2003)

Zimmerman, B. J., y Kitsantas, Handbook of competence and motivation, 1ㄹ Ed., 509-526, Guilford Press, New York, EE. UU (2005)

Zimmerman, B. J., y Moylan, A.R., Handbook of Metacognition in Education, 1를. Ed., 299-315, Routledge, New York (2009) 
